\title{
Determining the amount of Acrylamide in Potato Chips Using Xanthydrol as a Derivative Representative with Gas Chromatography-Mass Spectrometry
}

\author{
Maryam Zokaei ${ }^{1}$, Marzieh Kamankesh ${ }^{2}$, Saeideh Shojaei ${ }^{3}$, Abdorreza Mohammadi ${ }^{3 *}$ \\ 1- Master student of Food Biosciences and Technology, National Nutrition and Food Technology Research Institute, Faculty of Nutrition Sciences and \\ Food Technology, Shahid Beheshti University of Medical Sciences, Tehran, Iran. \\ 2- PhD student, Iran University of Science and Technology, Tehran, Iran \\ 3- Dept. of Food Science and Technology, National Nutrition and Food Technology Research Institute, Faculty of Nutrition Sciences and Food \\ Technology, Shahid Beheshti University of Medical Sciences, Tehran, Iran.
}

\section{A B S T R A C T}

Background and Objectives: In the production process of foods rich in carbohydrates and some other foods containing precursors of acrylamide formation, there is the possibility of acrylamide creation, which is a mutagen and carcinogen material. This study aims to introduce and optimize a new method for determining acrylamide in potato chips using xanthydrol as a derivative representative with gas chromatography-mass spectrometry.

Materials and Methods: Important factors in the derivatization and measurement processes were optimized using the one-factor-at-a-time method. The peak areas were assumed as the GC-MS response to evaluate the extraction efficiency of acrylamide, and optimization for all tests was performed two times. Figures of merit of the proposed method were evaluated. The amount of acrylamide in four potato chips samples, obtained from the market in Tehran city, was determined using the proposed method.

Results: The optimum amounts of effective parameters, including derivatization temperature at $25^{\circ} \mathrm{C}$, derivatization time $(40 \mathrm{~min})$, xanthydrol volume $(40 \mu \mathrm{L})$, extracted solvent volume $(500 \mu \mathrm{L})$ and $\mathrm{pH}=7$ were determined. The figures of merit for the proposed method were at the ideal range. The maximum and minimum amounts of acrylamide in the chip samples were also measured.

Conclusions: The performance and reliability of proposed method as a simple, efficient and rapid method for determining acrylamide in potato chip samples were demonstrated.

Keywords: Potato chips, Acrylamide, Xanthydrol, Gas Chromatography-Mass Spectrometry

\section{Introduction}

Acrylamide is a white water-soluble substance, which is solid at room temperature. Its carcinogenic feature has been approved in the past; this may result in chromosomal mutation in DNA and also damage to the nervous system $(1,2)$. Acrylamide is transferred to all parts of the body through the blood stream. It is found in many tissues and parts of the body such as liver, kidney, brain, heart and even breast milk (1). Since 2002 that the possibility of creation of this carcinogenic compound was proposed in carbohydrate-rich foods, it has been given special attention in terms of food security $(1,3)$.

Given the basic mechanism of acrylamide formation, reaction of reducing sugar carbonyl group with the amine group of some amino acids, particularly asparagine, the possibility of its creation in products rich in these precursors can be expected. Daily intake of acrylamide through diet in adults is about 0.3 to 0.6 microgram per kilogram, and in children and adolescents, is about 0.4 to $0.6 \mu \mathrm{g} \mathrm{kg}^{-1}$ 
may be due to more consumption of foods susceptible to acrylamide, particularly chips by these age groups $(4,5)$. Acrylamide exists not only in grain products such as bread, cake and biscuit but also in oil, nuts, meat, coffee, chocolate, dry milk, tea and drinking water. It has been shown that the amount of acrylamide in fried potato products, particularly chips, is in the highest range (1). The amount of acrylamide is different in various food products; it is $600 \mathrm{ng} \mathrm{g}^{-1}$ for chips.

By measuring the amount of acrylamide in food products, we can obtain the suitable conditions of thermal and production processes to produce safer products, and also have an estimation of its amount in the household's daily food basket. So far, various instrumental analytical methods (gas chromatography (GC), high-performance liquid chromatography (HPLC)) and immunological methods have been used for measuring acrylamide $(6,7)$. GC and HPLC are the major methods in terms of accuracy, repeatability and sensitivity $(1,6,8,9)$. Furthermore, they facilitate the sample preparation and thus are economic. Biological methods have disadvantages such as low repeatability and sensitivity, and are more qualitative. Liquid chromatography has high cost, high detection limit and performance problems, while GC has high repeatability, lower cost and high accuracy; this issue is considerable from the economic perspective and with respect to low or very low amount materials. Also due to high repeatability, more reliable data is obtained in GC analysis. The major weakness of this method is that due to non-volatility of acrylamide, the identification using GC needs to derivatization that is time consuming and makes the work complicated. Therefore, there should be a method that needs less and simpler stages of derivatization, and can makes GC appropriate and desirable for acrylamide identification.

Acrylamide derivatization using xanthydrol is a new method that has been given more consideration in recent years $(10,11)$, and compared with usual methods such as bromation derivatization, it is consists of much simpler and shorter steps. In addition, the chemicals used in bromation method are smelly and dangerous, while derivatization with xanthydrol is without these disadvantages (12). Foose in 1987 used xanthydrol to identify the primary amides and urea in the urine (13). Since then, xanthydrol has found an especial importance in the laboratory sciences, particularly for measuring protein in the urine. In recent years, it has been more considered for measuring amide compounds; this is the main reason for establishing balanced and normal laboratory conditions to work with xanthydrol. Optimum conditions for derivatization of acrylamide with bromide are high temperature and long time, while derivatization with xanthydrol compared with mentioned method requires less time and more balanced temperature (14). The aim of this study is to determe acrylamide, as well as optimizing the derivatization and measurement conditions of acrylamide in potato chips using xanthydrol as a derivative representative with gas chromatographymass spectrometry.

\section{Materials and Methods}

Chemicals and reagents: Chemical standards of acrylamide (99\%) and acetamide were purchased from Merck (Darmstadt, Germany). Hydrochloric acid, sodium chloride, ethanol, methanol, acetone, acetonitrile tetrachloroethylene, chloroform, carbon tetrachloride, dichloromethane, hydroxide potassium, xanthydrol, di-potassium hydrogen phosphate $\left(\mathrm{K}_{2} \mathrm{HPO}_{4}\right)$, potassium hexaferrocyanide (carrez I) and zinc acetate (carrez II) were also obtained from Merck (Darmstadt, Germany). For preparation of carrez solution I, $10.6 \mathrm{~g}$ of potassium hexaferrocyanide was dissolved in $100 \mathrm{~mL}$ distilled water. Carrez solution II was prepared by mixing $21.9 \mathrm{~g}$ of zinc acetate with 3 $\mathrm{mL}$ of acetic acid, then adjusting the volume to 100 $\mathrm{mL}$ with distilled water.

The primary standard solution of acrylamide and acetamide $\left(2000 \mu \mathrm{g} \mathrm{mL}^{-1}\right)$ was prepared in methanol. To obtain a working solution, the upper standard solution was diluted with methanol. Stock and working solutions were stored at $4^{\circ} \mathrm{C}$ in a refrigerator.

Instrumental conditions: Chromatographic separations and detections of the target analytes were performed using a 7890A GC system from Agilent Technologies (Palo Alto, CA, USA) with a triple-axis detector fitted with a split/split less injector and coupled with a 5975C inert MSD network mass selective detector. An HP-5 MS capillary column (5\% phenyl siloxane/95\% methyl polyorganosiloxane; 30 $\mathrm{m} \times 0.25 \mathrm{~mm}$ I.D., $0.25 \mu \mathrm{m}$ film thickness) was used 
for the separation of chemical compounds. The oven temperature was programmed as follows: $100^{\circ} \mathrm{C}$ held for $1 \mathrm{~min}$, ramped to $300^{\circ} \mathrm{C}$ at $20^{\circ} \mathrm{C} \mathrm{min}{ }^{-1}$, and held for $10 \mathrm{~min}$. Helium was used as a carrier gas in a constant flow of $0.8 \mathrm{~mL} \mathrm{~min}^{-1}$. The injector temperature and the auxiliary temperature were set at $290^{\circ} \mathrm{C}$ and $280^{\circ} \mathrm{C}$, respectively. Approximately $2 \mu \mathrm{L}$ of the sample was injected in a split mode with split ratio of 1:50. The compounds were quantified in the selected ion monitoring (SIM) mode, and one qualifier ion was selected for each compound.

Sample preparation: Three samples of chips were purchased from the Tehran city market. These samples were pulverized well. One gram of each sample was weighted and spiked with 100 and $50 \mathrm{ng}$ $\mathrm{g}^{-1}$ acetamide and acrylamide, respectively. In the next step, fat separation was performed using $3 \mathrm{~mL}$ of hexane, and then $7 \mathrm{~mL}$ distilled water was added and centrifuged at $4000 \mathrm{rpm}$ for $5 \mathrm{~min}$. Then the upper aqueous phase was separated, and $0.5 \mathrm{~mL}$ of carezz solution I (potassium hexaferrocyanide) and $0.5 \mathrm{~mL}$ of carrez solution II (zinc acetate) were added to precipitate the soluble carbohydrate and protein. This sample was thoroughly agitated, and then centrifuged for $5 \mathrm{~min}$ at $4000 \mathrm{rpm}$. Then the upper solution was separated. For derivatization process, $40 \mu \mathrm{L}$ of xanthydrol $5 \%$ in methanol and $2 \mathrm{~mL}$ of hydrochloric acid $\left(1 \mathrm{~mol} \mathrm{~L}^{-1}\right)$ were added to the sample solution and thoroughly shacked. This sample was kept at room temperature for $40 \mathrm{~min}$ to complete the derivatization step. After this stage, $0.5 \mathrm{~mL}$ of $\mathrm{KOH}$ $\left(2 \mathrm{~mol} \mathrm{~L}^{-1}\right)$ and $2 \mathrm{~mL}$ of $\mathrm{K}_{2} \mathrm{HPO}_{4}\left(2 \mathrm{~mol} \mathrm{~L}{ }^{-1}\right)$ were added to the sample solution. In this step, the $\mathrm{pH}$ of sample solution was 7. Finally, $500 \mu \mathrm{L}$ of chloroforms was added and mixed thoroughly and then centrifuged (4000 rpm for $5 \mathrm{~min}$ ). Finally, $1 \mu \mathrm{L}$ of the sedimented phase was directly injected into the GC-MS.

Statistical analysis: The most effective parameters on performing the extraction, including temperature of derivatization, derivatization time and xanthydrol concentration were chosen based on the literature and preliminary experiments. To optimize the values of these factors and to reach the best response, we used the one-factor-at-a-time method. Each of these factors was studied at five levels. For each of the three studied variables, high and low set points were selected. Two replicate extractions and quantitative determinations were performed for each experiment, and the peak areas were assumed as the GC-MS response to evaluate the extraction efficiency of acrylamide. Excel software was used to analyze the data and the design of the experiment.

\section{Results}

In the experimental procedure, some important variables including temperature of derivatization, derivatization time, xanthydrol concentration and $\mathrm{pH}$ usually affect on the extraction yield in analysis. Sample $\mathrm{pH}$ and extraction volume were selected according to our previous study (15-17). $\mathrm{pH} 7$ and $500 \mu \mathrm{L}$ chloroform were used for all experiments.

Derivatization reagent amount: Amount of derivatization reagent is another parameter that can affect on the derivatization and extraction processes. 40-120 $\mu \mathrm{L}$ of xanthydrol were selected for the derivatization step. $40 \mu \mathrm{L}$ of xanthydrol has the highest response; then it was employed as the best volume of derivatization reagent.

Time of derivatization: Derivatization time was one of the important factors that puts effect on the derivatization process. The time range of $20-60 \mathrm{~min}$ was evaluated. The results showed that the response was increased from 20 to $40 \mathrm{~min}$, and then remained unchanged (Figure 1). Therefore, $40 \mathrm{~min}$ was used as the best time for derivatization step.

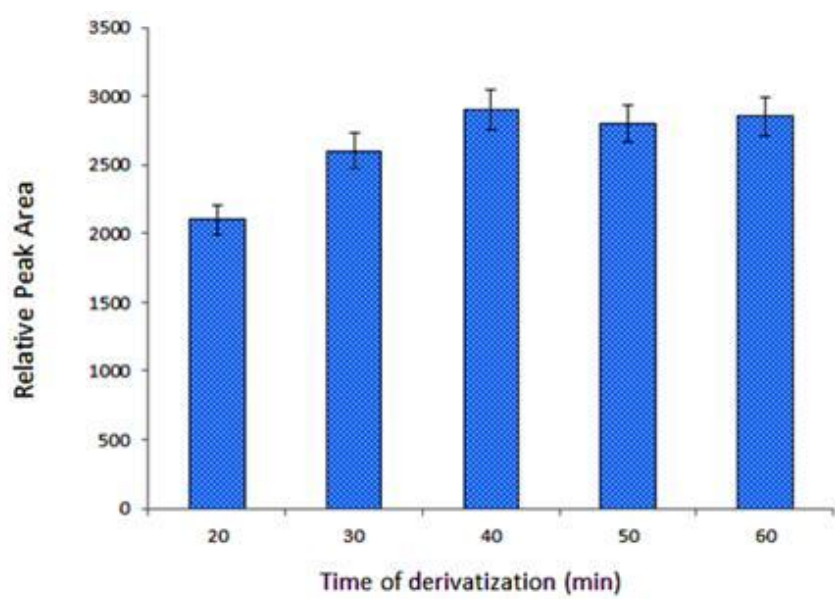

Figure 1. Effect of time of derivatization on the relative response factor $(\mathrm{n}=3)$.

Experimental conditions: temperature of derivatization: $25^{\circ} \mathrm{C}$; volume of the extraction solvent: $500 \mu \mathrm{L} ; \mathrm{pH}: 7$. 
Temperature of derivatization: In order to achieve appropriate temperature of derivatization, temperatures from $25^{\circ} \mathrm{C}$ to $60^{\circ} \mathrm{C}$ were tested. As shown in Figure 2, the ambient temperature $\left(25^{\circ} \mathrm{C}\right)$ is the best temperature in the derivatization step.

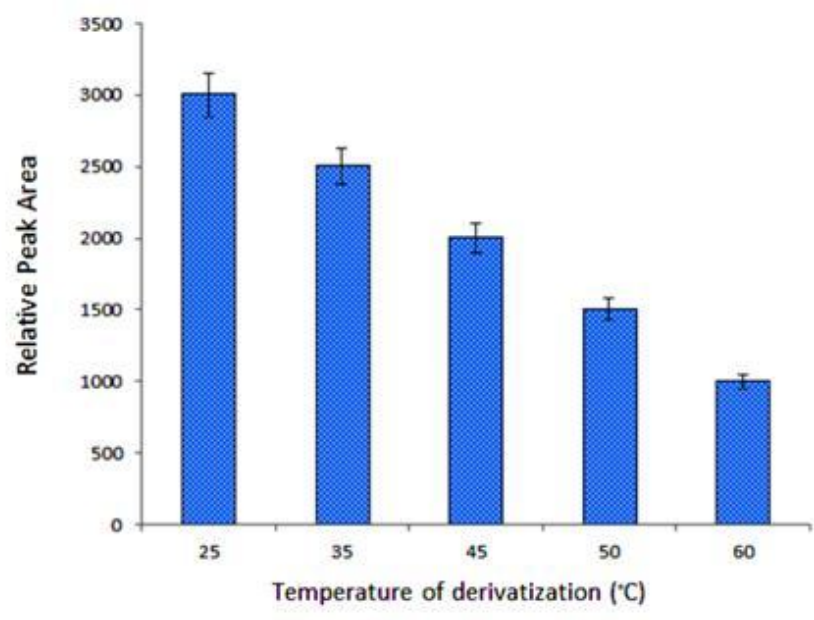

Figure 2. Effect of temperature of derivatization on the relative response factor $(n=3)$.

Experimental conditions: time of derivatization: $40 \mathrm{~min}$; volume of extraction solvent: $500 \mu \mathrm{L} ; \mathrm{pH}$ : 7 .
The figures of merit: In order to determine the figures of merit, parameters such as dynamic liner range (DLR), repeatability (RSD), limit of detection (LOD), limit of quantitation (LOQ) and enrichment factor $(\mathrm{EF})$ were investigated. The calibration curve was linear between 10 and $500 \mathrm{ng} \mathrm{mL}^{-1}$ with the coefficient determination $\left(\mathrm{R}^{2}\right)$ higher than 0.9993 . RSD percentage was calculated by the analysis of 7 repetitive analyses and was obtained as $6.8 \%$. The recovery and enrichment factors were 192 and $98 \%$, respectively. LOD and LOQ were obtained as $0.6 \mathrm{ng}$ $\mathrm{g}^{-1}$ and $2.0 \mathrm{ng} \mathrm{g}^{-1}$, respectively.

The method evaluation on real samples: The practical applicability of the proposed method was evaluated under the optimum conditions for the analysis of acrylamide. Five potato chips samples were purchased from the market in Tehran city. The results are reported in Table 1.

The concentrations of acrylamide in the potato chips samples were determined by the standard addition method. The obtained chromatogram of a real sample is shown in Figure 3.

Table 1. Acrylamide contents $\left(\mathrm{ng} \mathrm{g}^{-1}\right)$ obtained in the analysis of four potato chips samples using proposed method

\begin{tabular}{ccccc}
\hline Sample & Acrylamide concentration & Added amount & Analyzed amount & Recovery (\%) \\
\hline 1 & $89.20 \pm 6.06$ & 50.0 & $136.40 \pm 9.27$ & 98 \\
2 & $94.21 \pm 6.40$ & 50.0 & $139.84 \pm 9.50$ & 97 \\
3 & $68.04 \pm 4.62$ & 50.0 & $116.81 \pm 7.94$ & 99 \\
4 & $70.30 \pm 4.78$ & 50.0 & $117.90 \pm 8.01$ & 98 \\
\hline
\end{tabular}
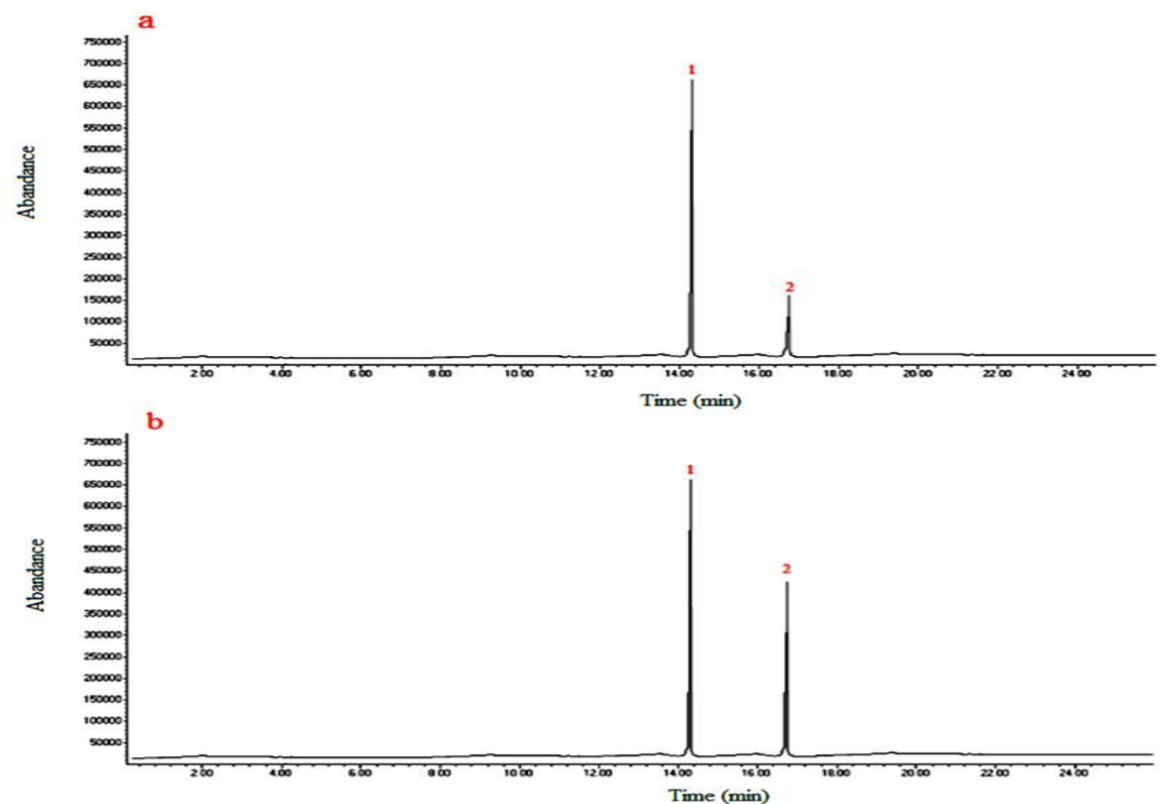

Figure 3. The chromatogram obtained by the proposed method for a potato chips sample under optimum conditions. (a) nonspiked and (b) spiked with $50 \mathrm{ng} \mathrm{g}^{-1}$ of acrylamide. 1) acetamide (internal standard), 2) acrylamide. 


\section{Disc ussion}

Analysis of acrylamide in the potato chips samples is problematic because of their extremely low concentrations and complexity of the matrix sample. Also analysis of acrylamid with GC requires derivatization step; therefore, it is considered as the most critical step in the overall analytical process. The optimum xanthydrol solution for derivatization has been considered by several researches $(18,19)$. In our research, the best result was achieved when we used $40 \mu \mathrm{L}$ xanthydrol; in more amounts, we had problem due to a white cloudy condition that made noise in the next steps of the operation. For temperature optimization, the best result was seen at ambient condition $\left(25^{\circ} \mathrm{C}\right)$, showing that xanthenacrylamide made from derivatization is so sensitive to heat. Some research has reported the same result; however, in another study, $60^{\circ} \mathrm{C}$ was reported as the best temperature for derivatization.

Regarding the time of derivatization, the results showed the $40 \mathrm{~min}$ has the best response. Times over $40 \mathrm{~min}$ showed reduction in response that can be related to sensitivity of acrylamide to heat. Similar results have been reported in some other articles (2023).

Validation: Analysis of acrylamide showed a linear relationship with high linear regression coefficients of determination for acrylamide $\left(\mathrm{R}^{2}>0.9993\right)$. The complete description of standard linearity supported by regression data is shown in Table 1 . The results indicated that the developed extraction method provided reasonably good accuracy for the analysis of acrylamide in the potato chips samples.

Real sample analysis: Potato chips samples were purchased from the Tehran city market and tested with the proposed method to evaluate the reliability of the developed technique. The concentrations of the analyte were calculated from the linear regression equations of the standard curves. The results are shown in Table 1. A clean separation and good chromatogram are readily achieved without the presence of matrix interference (Figure 3).

Conclusion: In this study, a fast, easy, reliable and effective method was developed to determine acrylamide in potato chips samples using xanthydrol derivatization. The effective parameters in derivatization of this method were optimized. The results showed that the carrez solution has a positive effect on the sediments of impurities. The advantages of this method compared with other available derivatization methods in acrylamide measurement are fastness, highly sensitivity, good precision and reliability of the analysis. The developed method was applied for the trace determination of acrylamide in potato chips samples, and satisfactory results were obtained.

\section{Financial disclosure}

The authors declared no financial interest.

\section{Funding/Support}

This study was supported by National Nutrition and Food Technology Research Institute.

\section{References}

1. Hogervorst JG, Schouten LJ, Konings EJ, Goldbohm RA, van den Brandt PA. A prospective study of dietary acrylamide intake and the risk of endometrial, ovarian, and breast cancer. Cancer Epidemiol Biomarkers Prevention 2007;16:2304.

2. Stadler RH, Lineback DR. Process-induced food toxicants: occurrence, formation, mitigation, and health risks: John Wiley \& Sons; 2008.

3. Tareke E, Rydberg P, Karlsson P, Eriksson S, Törnqvist M. Analysis of acrylamide, a carcinogen formed in heated foodstuffs. J Agricult Food Chem 2002;50(17):4998-5006.

4. Tardiff RG, Gargas ML, Kirman CR, Carson ML, Sweeney LM. Estimation of safe dietary intake levels of acrylamide for humans. Food Chem Toxicol 2010;48(2):658-67.

5. Mottram DS, Wedzicha BL, Dodson AT. Food chemistry: Acrylamide is formed in the Maillard reaction. Nature 2002;419(6906):448-9.

6. Claus A, Carle R, Schieber A. Acrylamide in cereal products: A review. J Cereal Sci 2008;47(2):118-33.

7. Boroushaki MT, Nikkhah E, Kazemi A, Oskooei M, Raters M. Determination of acrylamide level in popular Iranian brands of potato and corn products. Food Chem Toxico 2010;48(10):2581-2584.

8. Oracz J, Nebesny E, Żyżelewicz D. New trends in quantification of acrylamide in food products. Talanta 2011;86:23-34.

9. Hu Q, Xu X, Li Z, Zhang Y, Wang J, Fu Y, et al. Detection of acrylamide in potato chips using a fluorescent sensing method based on acrylamide polymerization-induced distance increase between 
Maryam Zokaei, et al: Determination of acrylamide in potato chips with gas chromatography- mass spectrometry

quantum dots. Biosens Bioelectron 2015 4/15/;54(0):64-71.

10. Hitzeman R, Chinault A, Kingsman AJ, Carbon J, editors. Detection of E. coli clones contaning specific yeast genes by immonological screening. ICN-UCLA Symposium Mol Cel Biol 2012.

11. Wenzl T, De La Calle MB, Anklam E. Analytical methods for the determination of acrylamide in food products: a review. Food Addit Contamin. 2003 20(10):885-902.

12. Phillips R, Pitt BM. The use of Xanthydrol as a reagent for the characterization of primary amides. J Am Chem Soc 1943;65(7):1355-7.

13. Yamazaki K, Isagawa S, Kibune N, Urushiyama T. A method for the determination of acrylamide in a broad variety of processed foods by GC-MS using xanthydrol derivatization. Food Addit Contam: Part A 2012;29(5):705-15.

14. Friedman M. Chemistry, biochemistry, and safety of acrylamide. A review. J Agricult and Food Chem 2003;51(16):4504-26.

15. Kamankesh M, Mohammadi A, Hosseini H, Tehrani ZM. Rapid determination of polycyclic aromatic hydrocarbons in grilled meat using microwave-assisted extraction and dispersive liquid-liquid microextraction coupled to gas chromatography-mass spectrometry. Meat Sci 2015; 103: 61-67.

16. Ramezani H, Hosseini $H$, Kamankesh $M$, Ghasemzadeh-Mohammadi V, Mohammadi A. Rapid determination of nitrosamines in sausage and salami using microwave-assisted extraction and dispersive liquid-liquid microextraction followed by gas chromatography-mass spectrometry. Eur Food Res Technol 2014; 240(2): 441-450.

17. Ramezani H, Kamankesh M, Hoseiny H, Ghasemzadeh V, Mohammadi A. Microextraction and determination of nitrosamines in sausage and salami in Tehran market using gas chromatography-mass spectrometry. Iranian J Nutr Sci Food Technol 2014; 9(1): 57-67.

18. Phillips R, Pitt BM. The use of Xanthydrol as a reagent for the characterization of primary amides. J Am Chem Soc 1943;65(7):1355-7.

19. -www.jbc.org/content/220/2/957.full.pdf

20 .YW. Fast and simple screening for the simultaneous analysis of seven metabolites derived from five volatile organic compounds in human urine using on-line solidphase extraction coupled with liquid chromatographytandem mass spectrometry. Talanta 2015;132:469-78.

21. Bogo V, Hill TA, Young RW. Comparison of accelerod and rotarod sensitivity in detecting ethanol-and acrylamide-induced performance decrement in rats: Review of experimental considerations of rotating rod systems. Neurotoxicology 1981;2(4):765-87.

22. Ghasemzadeh V, Atefi M, Homaunfar R, Hejazi E, Todeh Rosta Z. Investigation of Acrylamide formation and reduction ways of it in specific food products. Iranian J Nutr Food Sci Res 2013;7(5): 957-968 [in persion].

23. Koushki MR, Mohammadian M, Koohy-Kamaly P. Effective ways of reducing acrylamide formation in fried potato products. Iranian J Nutr Sci Food Technol 2013;7(5): 939-947 [in Persion]. 